\title{
An Adaptive Neighbourhood Structuring Element Based Enhancement Technique for Nuclear Images
}

\author{
Neethu M. Sasi and Jayasree V. K.
}

\begin{abstract}
The application of radioactive isotopes for imaging human body parts developed into nuclear imaging technology, including single photon emission computed tomography imaging (SPECT). Visual inspection and interpretation of SPECT images is a challenging task as there is random scattering of photons during the image reconstruction process which affects its contrast. Recognizing even gradual changes in color intensity aids in the interpretation of images. The paper proposes a new image processing technique to enhance cardiac nuclear images generated by a single photon emission computed tomography device by improving the contrast features. The method utilizes the concept of adaptive techniques in morphology. As an initial step, color space conversion is performed to convert the image to a color space suitable for its processing and each slice or tile corresponding to the gated cardiac cycle is extracted. In the second step, the size of the neighbourhood area is selected for the operation of the structuring element. Morphological processing is done as a final step. This methodology can be used as a pre-processing module for computer aided diagnostic systems. The proposed method employs a novel image dependent technique for the enhancement of cardiac SPECT images making use of morphology in a suitable color space. Qualitative and quantitative evaluations prove the effective performance of the proposed algorithm in enhancing cardiac SPECT images.
\end{abstract}

Index Terms-Morphological processing, nuclear medicine imaging, positron emission tomography, single photon emission computed tomography.

\section{INTRODUCTION}

Medical imaging is a critical tool in the hands of bio-medical informatics for the correct prognosis and diagnosis of maladies affecting human body. The use of gamma rays for diagnostic purposes started the era of nuclear medicine in medical imaging techniques, opening up a new frontier as one of the latest advancements in the field of medical imaging [1]. Every imaging technique with an output in digital form has been effectively using computational techniques for improving the quality of its image output. In this study we propose a simple and straightforward method using morphology for improving the quality of nuclear medicine images. A nuclear medicine physician visually analyses the SPECT images and interprets them, guided by the variations in color intensity (in the case of color images) or gray scale intensity (in the case of gray scale images). Physicians prefer color images for visual interpretation as color is better for identifying small defects and leads to better inter-observer agreement. The approach proposed by this

Manuscript received on June 26, 2015; revised November 8, 2015.

The authors are with the Department of Electronics Engineering, Model Engineering College, Cochin University of Science and Technology, India (e-mail: neethumsasi@gmail.com, jaya@mec.ac.in). paper is unique in its use of adaptive neighborhood for the processing of different image regions in an identified color space.

The information generated from nuclear imaging techniques provide data on the structure as well as the function of the organ being imaged [2] and [3]. To perform nuclear medicine imaging, a small amount of a radioactive tracer is injected into the patient's body, which is taken up by different organs in proportion to blood flow. The tracer amount in the organ of interest is then assessed by imaging the patient with a gamma camera which is capable of detecting the small amount of radiation emitted by the tracer. Both gray scale and color images can be generated by nuclear imaging systems. The different gray levels in a gray scale image or different colors in a color image represent different amounts of tracer uptake.

A singular application of nuclear medicine imaging technique is in the study of cardiac ailments since it could be used as a non intrusive method of diagnosis. Planar imaging, Single Photon Emission Computed Tomography (SPECT) and Positron Emission Tomography (PET) are the main nuclear medicine imaging modalities. Planar imaging gives a two dimensional view of the three dimensional distribution of radioactive tracer. Both SPECT and PET uses camera to acquire multiple two dimensional images from multiple directions which are then used to reconstruct three dimensional datasets. In SPECT the tracer emits gamma radiation that is measured directly and in PET the tracer emits positrons, which cause two gamma photons to be emitted in opposite directions. SPECT and PET differ in the radioactive tracer used which affects the sensitivity of the detection methods.

SPECT technique is cheaper as compared to PET, but SPECT images are more blurred than PET images [4] and [5]. Factors like limited number of counts and scatter of photons contribute to the degradation of SPECT images. By limiting this image degradation factor, a precise knowledge on the nature of blood flow could be obtained, serving as the primary guideline in the planning of post diagnostic procedures. If we could improve the quality of SPECT image by image enhancement techniques, it would deliver better information, both visually and for computer aided diagnosis, at a much cheaper cost as compared to PET. The novel method proposed by this work would enhance the quality of SPECT image using image processing techniques.

The literature describes different algorithms for image enhancement, which results in modified image output with more visual information. Being a relatively new technology in medical imaging applications, the related studies and literature resources in the field of nuclear medicine image enhancement are limited. The in-depth studies and the literature already available in image enhancement techniques 
are largely limited to the domain outside nuclear imaging.

A technique which automatically extracts nuclear boundaries by segmentation using thresholding and region growing has been explained by Wang et al. in [6]. Falk et al. proposes a method for the enhancement of noisy nuclear images using mean field annealing [7]. In both [6] and [7] the work has been accomplished on planar images. A similar method has been proposed in [8] for the enhancement of gated cardiac nuclear images. It uses a combination of mean field annealing and gradient edge detection and the work has been done on images in gray scale.

Further studies include an enhancement methodology for PET images [9], where the approach is based on the combination of wavelet transform and curvelet transform. The use of wavelet transform in nuclear medicine image processing is employed in [10]-[12]. The main issue in using wavelet transforms lies in the selection of a proper wavelet. In [13] principal component analysis is used for the enhancement of original scintigraphic image sequence. An enhancement using segmentation based on a multi resolution elliptical coordinate transformation and dynamic contour tracking has been proposed in [14]. An enhancement framework utilizing the powerfulness of Bayesian model and complex wavelet packets with a pre-processing stage of Anscombe transformation is detailed in [15]. The shortcoming is that the effectiveness of the above method is evaluated using only planar scintigraphic images.

A considerable majority of the literature resources related to the enhancement of nuclear medicine images are further restricted down to studies where images are in gray scale. In this work we propose an algorithm for enhancing cardiac SPECT images in gray scale as well as color scale. In the proposed scheme a novel approach has been used, in which, morphological operations on image regions of smaller dimension in a suitable color space using adaptive structuring elements is employed. The rest of the paper is organized as follows. Section II describes the methods in detail. Preliminary concepts are given and then a detailed description of the proposed method is given. Section III evaluates the performance of the proposed method both qualitatively and quantitatively. The proposed algorithm is also compared with other existing techniques. Section IV concludes the paper.

\section{METHODS}

The proposed method works in three main stages as shown in Fig. 1 - separation of chrominance channels and extraction of sub regions or tiles or slices from each chrominance channel, selection of the size of the structuring element suitable for each tile adaptively and morphological processing of each tile with the corresponding structuring element. A cardiac nuclear image consists of sub regions or tiles or slices which show the functioning of the heart at different instances of time. Each tile shows the amount of blood in the left ventricle at a particular instant of heart cycle. The tiles are extracted using image processing techniques and further processing is done on individual tile. The method has been applied to 40 different cardiac nuclear images in RGB color space obtained from a two collimator single photon emission computed tomography device. The images are taken from 40 different patients in the age group of 40 to 65 years, with 17 patients having normal functioning of heart and 23 patients having myocardial perfusion defects. The relative differences in the uptake of tracer between myocardial pixels are represented in the image as a change in color. Institutional review board approval was obtained for the data collection in this study and waived the need for informed consent since the data does not reveal the patient identity.

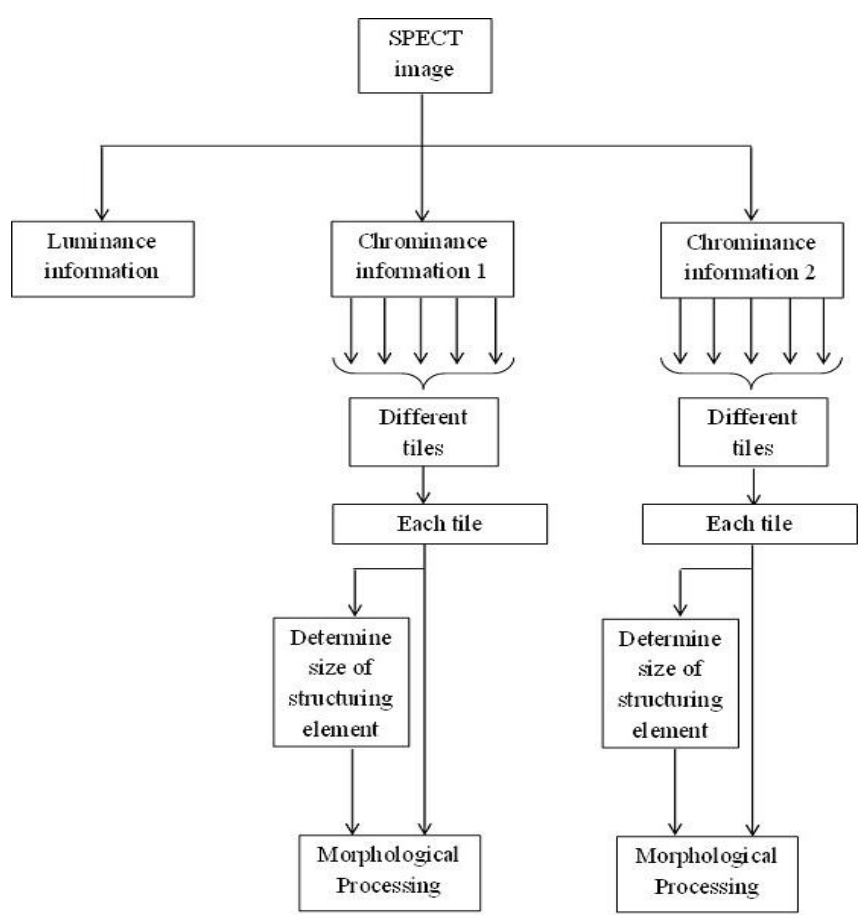

Fig. 1. Block diagram showing the basic stages in the proposed method.

\section{A. Preliminary Concepts in Morphology}

Morphological image processing is an important tool for image enhancement. It involves the use of mathematical morphology for describing quantitatively the geometrical information of image objects. Morphology was introduced in the late 1960's [16] and since then it has been used in the field of image processing for various applications. Mathematical morphology is expressed in terms of set theory. When mathematical morphology is applied to image processing, the image and the objects in the image are treated as sets. An image $A$ is represented by a two dimensional set of pixels in a rectangular format where, $A(i, j)$ is a pixel at coordinate $(i, j)$ in the image $A$. In morphological image processing the image is processed by a series of morphological operations using another set known as a structuring element which exploits the geometrical information in the image. The structuring element $S$ is also represented by a two dimensional set of pixels. The basic morphological operations are dilation, erosion, opening and closing. The dilation of a binary image $A$ by the structuring element $S$, where $A$ and $S$ are members of the two dimensional integer space $Z_{2}$, is given by

$$
\begin{gathered}
A \oplus S=\left\{z[\hat{S}]_{z} \cap A \neq \phi\right\} \\
\left.A \oplus S=\left\{z \mid[\hat{S}]_{z} \cap A\right] \subseteq A\right\}
\end{gathered}
$$


where

$$
\hat{S}=\{w \mid w=-s, s \in S\}
$$

and

$$
[\hat{S}]_{z}=\left\{c \mid c=s^{\prime}+z, s^{\prime} \in \hat{S}\right\}
$$

The erosion of $A$ by $S$ is

$$
A \Theta S=\left\{z[S]_{z} \subseteq A\right\}
$$

The opening and closing of set $A$ by the structuring element $S$ is defined in (6) and (7) respectively.

$$
\begin{aligned}
& A \circ S=(A \Theta S) \oplus S \\
& A \bullet S=(A \oplus S) \Theta S
\end{aligned}
$$

The dilation of the gray scale image $A$, with domain $D_{A}$, by the structuring element $S$, with domain $D_{S}$, is

$$
(A \oplus S)(p, q)=M_{\text {max }}
$$

where $M_{\max }$ is the maximum value of $f(p-x, q-y)+S(x$, $y) /(p-x),(q-y) € D_{A} ;(x, y) € D_{S}$

The erosion of the gray scale image $A$, with domain $D_{A}$, by the structuring element $S$, with domain $D_{S}$, is

$$
(A \Theta S)(p, q)=N_{\min }
$$

where $N_{\min }$ is the minimum value of $f(p+x, q+y)-S(x$, $y) /(p+x),(q+y) € D_{A} ;(x, y) € D_{S}$

\section{B. Proposed Method}

\section{1) Separation of chrominance channels}

The performance of basic edge detectors on cardiac SPECT images is studied in different color spaces to establish a suitable color space for its processing [17]. Since the image under consideration contains predominantly red and blue components, the operation in $\mathrm{YCbCr}$ color space gives the best result. In this color space, $C b$ stores the information about blue component and $\mathrm{Cr}$ stores the information about red component [18].

The image is converted into the color space with one luminance, $Y$, and two chrominance, $C b$ and $C r$, components from red $R$, blue $B$ and green $G$ components using the relation given in (10).

$$
\left[\begin{array}{c}
Y \\
C b \\
C r
\end{array}\right]=\left[\begin{array}{c}
16 \\
128 \\
128
\end{array}\right]+\left[\begin{array}{ccc}
65.48 & 128.55 & 24.97 \\
-37.80 & -74.20 & 112.00 \\
112.00 & -93.79 & -18.214
\end{array}\right] X\left[\begin{array}{c}
R \\
G \\
B
\end{array}\right]
$$

The two chrominance channels are to be separated out and each channel needs to be divided into many tiles, $T_{c b i}$ and $T_{c r i}$, each with lower dimension. If the images are in gray scale the whole image itself is divided into many tiles, $T_{g i}$, each with lower dimension.

\section{2) Adaptive selection of the size of the structuring} element

Each tile is then processed morphologically by using structuring elements which are chosen adaptively. The structuring element keeps moving through the tile and on its way, operates on pixels in a predefined neighbourhood. If the size of this neighbourhood area is chosen as the same for every tile throughout the image, certain intricate portions may not be processed as required. Since cardiac nuclear images depict the pumping action of heart, the nature of each image tile will be different depending on the amount of blood contained in the heart at that time. In order to prevent over enhancement and under enhancement of image regions, the tiles are processed using structuring elements whose sizes depend on its nature. An analysis of pixels is made for each tile to get an idea about the amount of blood flow and the size of the structuring element is chosen accordingly to give a better result. Analysis is done using binary thresholding. Each tile is converted into a binary image. The output binary image has values of 1 (white) for all pixels in the input image with intensity greater than a given level (for representing the blood flow) and 0 (black) for all other pixels. Fig. 2 shows the original image and the binary thresholded image.

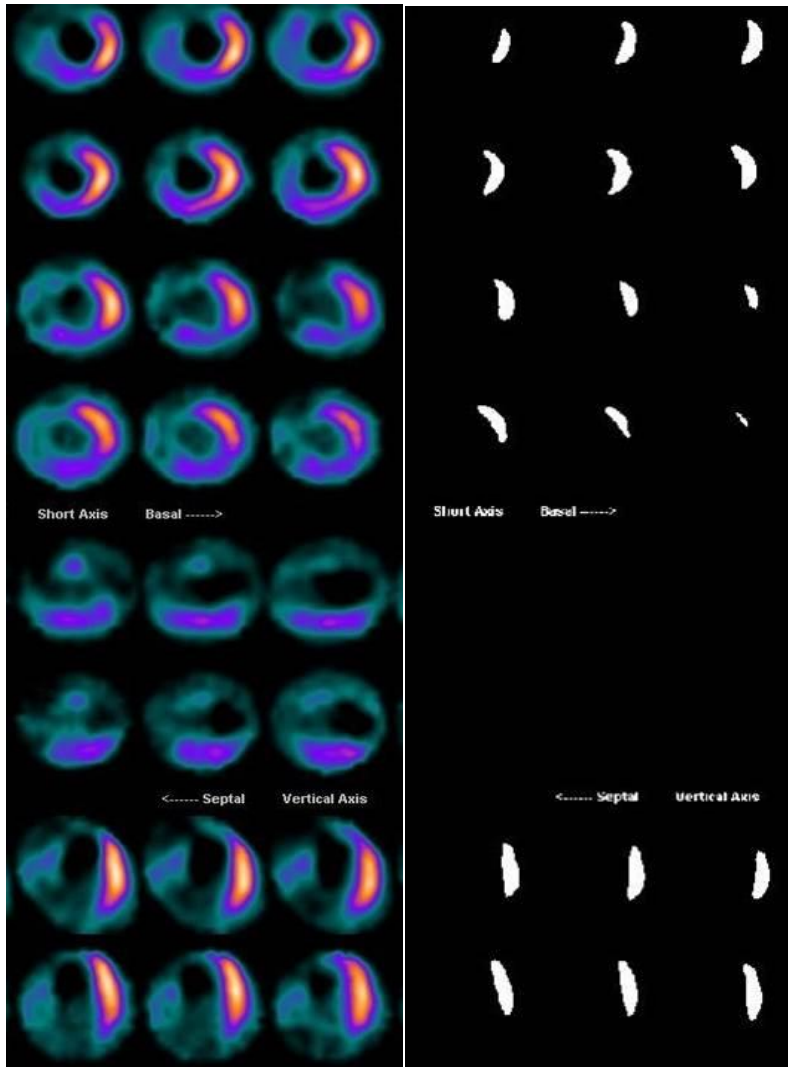

Fig. 2. SPECT image and thresholded image.

The binary thresholded image is analysed to get an estimate of the pixel count corresponding to the blood flow. The size of the structuring element is chosen such that the tiles with lower pixel count are processed in a bigger neighbourhood. This helps in enhancing even the smaller features in the image. At the same time over enhancement of tiles with higher pixel count is taken care of by choosing smaller neighbourhood.

\section{3) Morphological processing}

Opening and closing are morphological operations that operate on features smaller than the size of the structuring element. Morphological processing is carried out in two stages. In the first stage a morphologically opened image tile 
for each channel, denoted by $T_{c b i-o}$ and $T_{c r i-o}$, is subtracted from the original image tile to get pixels, denoted by $T_{c b i-t h}$ and $T_{\text {cri-th }}$, that are removed by opening. In the case of gray scale images the respective tiles are denoted by $T_{g i-o}$ and $T_{g i-t h}$.

$$
\begin{gathered}
T_{c b i-o}=\left(T_{c b i} \Theta S\right) \oplus S \\
T_{c b i-t h}=T_{c b i}-T_{c b i-o} \\
T_{c r i-o}=\left(T_{c r i} \Theta S\right) \oplus S \\
T_{c r i-t h}=T_{c r i}-T_{c r i-o} \\
T_{g i-o}=\left(T_{g i} \Theta S\right) \oplus S \\
T_{g i-t h}=T_{g i}-T_{g i-o}
\end{gathered}
$$

This process extracts light features that are smaller than the structural elements. These pixels are then added to the original image tile to enhance the contrast.

In the second stage the original image tile is subtracted from a morphologically closed image, denoted by $T_{c b i-c}$ and $T_{c r i-c}$, to get pixels which represent intensity troughs in the image tile, denoted by $T_{c b i-b h}$ and $T_{c r i-b h}$. In gray scale images these tiles are denoted by $T_{g i-c}$ and $T_{g i-b h}$ respectively.

$$
\begin{aligned}
& T_{c b i-c}=\left(T_{c b i} \oplus S\right) \Theta S \\
& T_{c b i-b h}=T_{c b i-c}-T_{c b i} \\
& T_{c r i-c}=\left(T_{c r i} \oplus S\right) \Theta S \\
& T_{c r i-b h}=T_{c r i-c}-T_{c b i} \\
& T_{g i-c}=\left(T_{g i} \oplus S\right) \Theta S \\
& T_{g i-b h}=T_{g i-c}-T_{g i}
\end{aligned}
$$

This process extracts dark features which are then subtracted from the contrast enhanced image.

\section{RESULTS AND DISCUSSION}

The performance of the proposed algorithm is evaluated both qualitatively and quantitatively.

\section{A. Qualitative Evaluation}

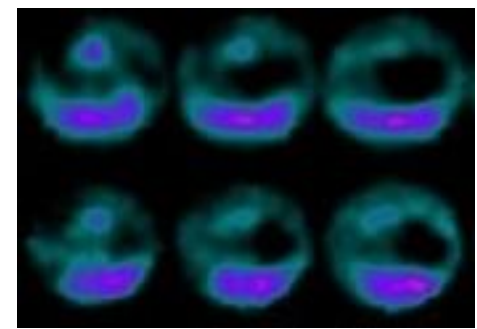

Fig. 3. SPECT image (low count area).

This section of the paper evaluates the application of the proposed method to cardiac nuclear images obtained from a two collimator single photon emission computed tomography device qualitatively. Fig. 3 and Fig. 5 show original SPECT images corresponding to low blood count and high blood count respectively. Fig. 4 and Fig. 6 show the respective enhanced images.

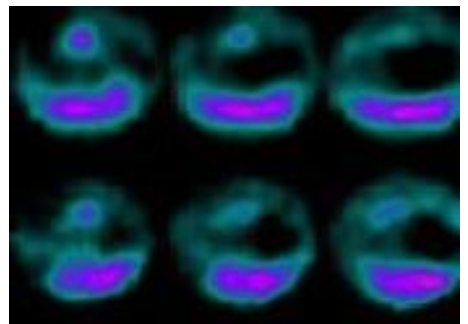

Fig. 4. Enhanced SPECT image (low count area).
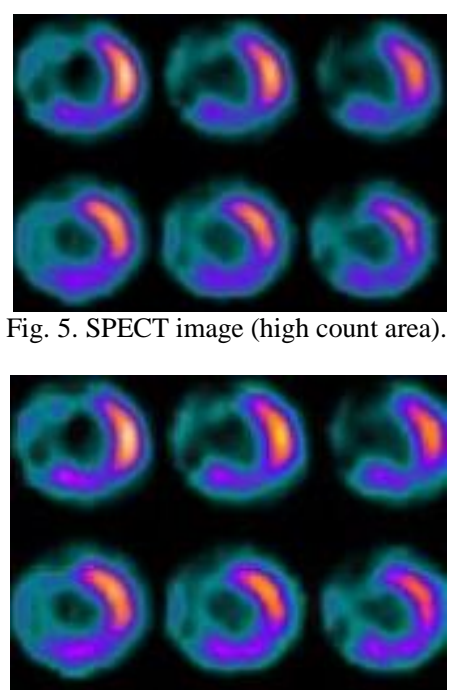

Fig. 6. Enhanced SPECT image (high count area).

The resultant image has registered an improvement in terms of its contrast, without loss in information as seen in Fig. 7 and Fig. 8.

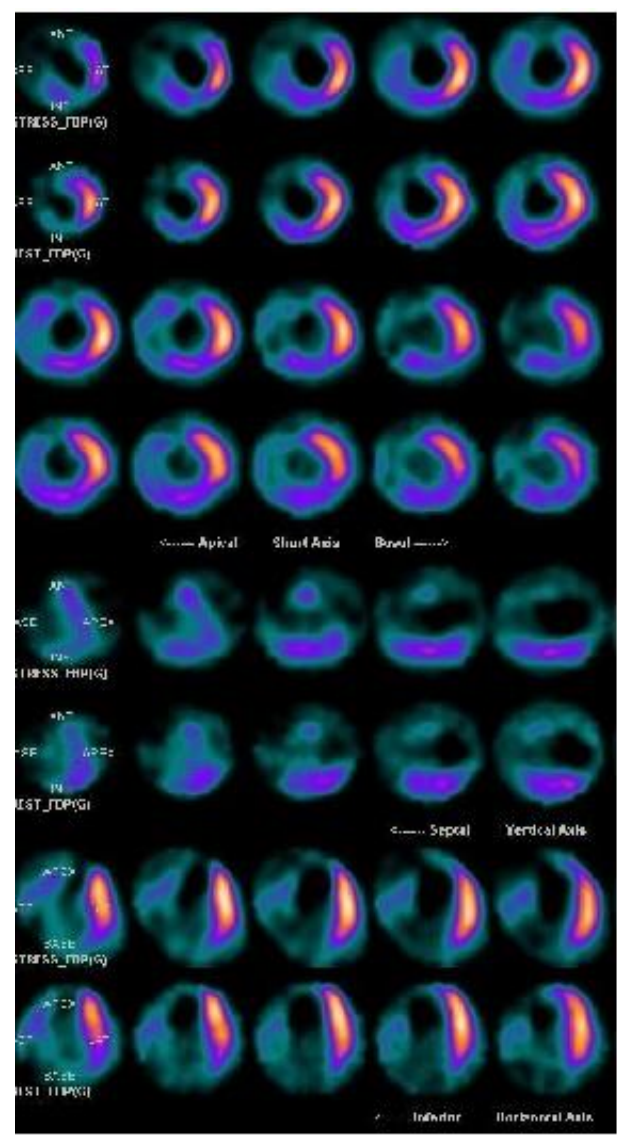

Fig. 7. Original SPECT image. 
The algorithm is also tested on gray scale images and the corresponding input and output images are in Fig. 9.

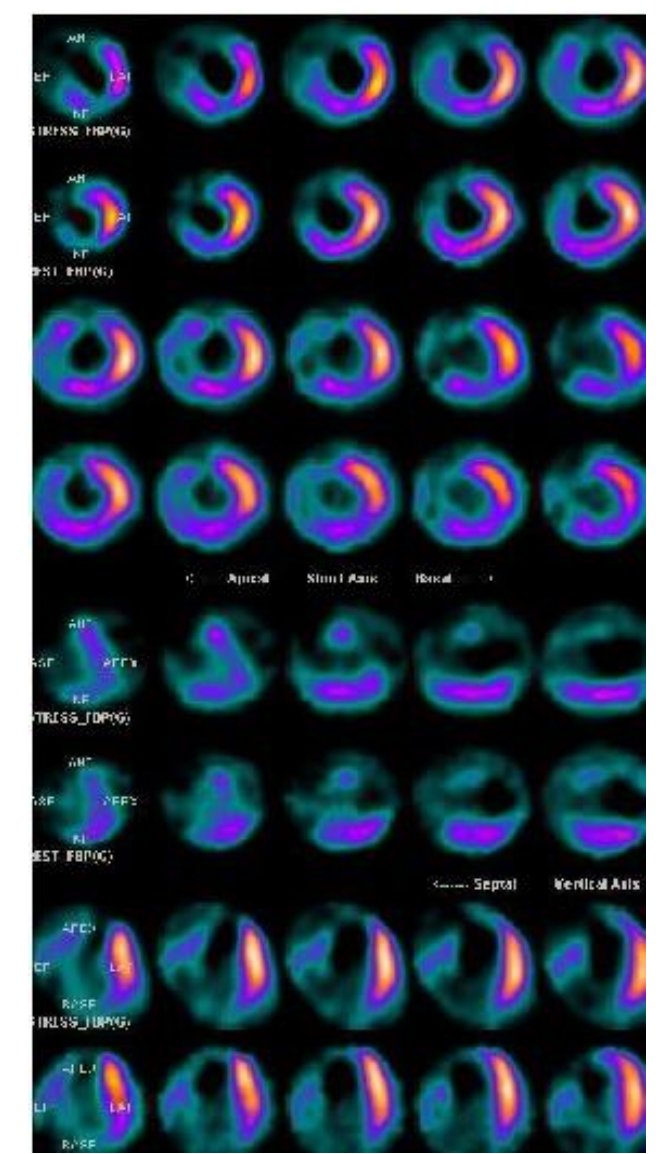

Fig. 8. SPECT image enhanced using the proposed method.

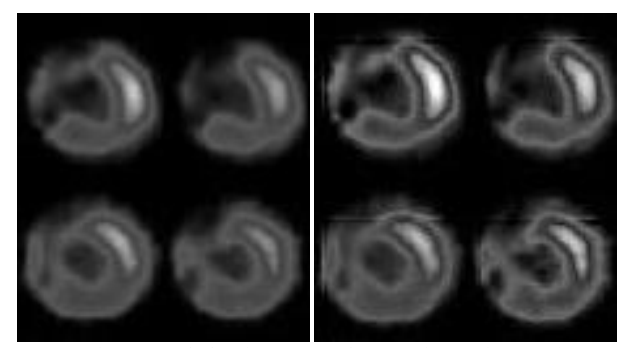

Fig. 9. In gray scale. a) Original SPECT image b) Enhanced SPECT image.

\section{B. Quantitative Evaluation}

Simulations have been carried out to evaluate the performance of the proposed scheme quantitatively. 40 real cardiac images, taken from 40 different patients, have been used to prove the efficiency of the proposed method in terms of contrast improvement and validation of trueness to the original image information. 17 patients have normal functioning of the heart and 23 patients have myocardial perfusion defects. $960 \times 510$ images, each with 40 slices or tiles are used for the experiments. As a final step, evaluation against existing enhancement techniques is also done quantitatively.

\section{1) Evaluation of contrast enhancement}

Factors like limited number of counts and scatter of photons mainly contribute to the degradation of light features in SPECT images when compared to the dark features. Decreased contrast results in poorer visibility of fine details in the image. Contrast enhancement of such light features helps in improving the quality of images to a great extent.
The increase in contrast is measured using Michelson's formula [19] which is considered the best option for the images where dark and light features take up similar fractions of area. The application of the formula gives an average increase in contrast of $15 \%$ for the enhanced image over original image.

The image pixels corresponding to the blood flow in left ventricular region for original SPECT, enhanced SPECT and original PET images are shown in Fig. 10. Both the original SPECT and original PET images are acquired from the same patient at the same instant of time. The normalized image pixel values corresponding to the left ventricular region for original SPECT, enhanced SPECT and original PET images are compared graphically in Fig. 11. The visual as well as the graphical evaluation proves the success of the proposed method in achieving a post enhancement image quality above the original SPECT image, but slightly below-par the equivalent PET sample.

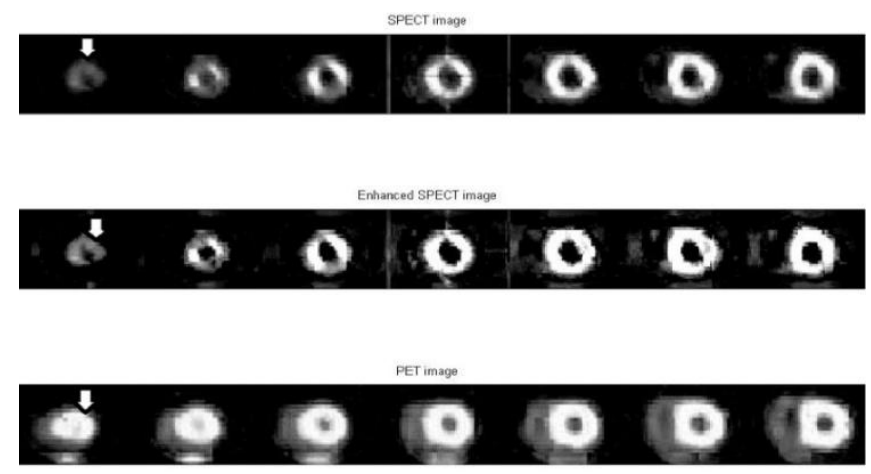

Fig. 10. Pixels showing blood flow in left ventricle for SPECT, enhanced SPECT and PET images.

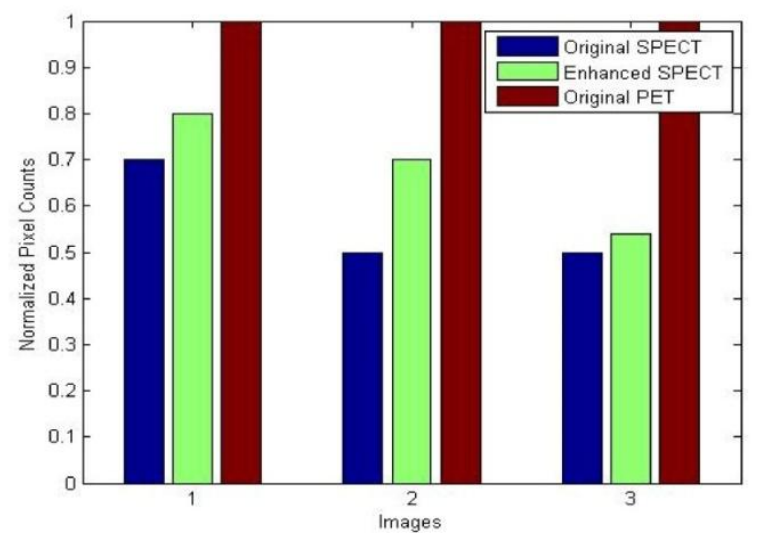

Fig. 11. Comparison of pixel counts representing blood flow in left ventricle for SPECT, enhanced SPECT and PET images.

2) Evaluation for validation of trueness of the enhanced image with respect to the original image

TABLE I: PAIRED SAMPLES T TEST RESUltS

\begin{tabular}{ccccc}
\multicolumn{5}{c}{ TABLE I: PAIRED SAMPLES T TEST RESULTS } \\
\hline \hline Mean & Standard Deviation & $\mathrm{t}$ & $\mathrm{df}$ & Sig (2 tailed) \\
\hline-0.00001 & 0.00293 & -0.023 & 34 & 0.982 \\
\hline \hline
\end{tabular}

A most commonly reported quantitative measure obtained for cardiac SPECT images is left ventricular ejection fraction (LVEF). It is calculated from the volume of blood in the left ventricle, which in turn, is computed from the pixel counts. If these pixel counts for the enhanced image stay the same or within a statistical acceptance value, the method can be validated for its trueness to the original image data. The 
statistical method of 'paired samples $t$ test', run in SPSS software has been used in validating the results as shown in Table I.

\section{3) Evaluation against existing comparable enhancement techniques}

In [20] and [21] the authors have presented the efficiency of contrast limited adaptive histogram equalization (CLAHE) for enhancing medical images. Table II presents some comparison results of the proposed method versus contrast limited adaptive histogram equalization method in color space. The results show that the proposed method leads to an improvement in contrast. It is worth a note that this is achieved without changing the information content of the original medical image as indicated by the mean square error (MSE) value and entropy difference value in the table. The MSE value indicates the mean square error in the pixel count between the original image and the enhanced image, that contributes to the calculation of ejection fraction. This must be minimum to ensure that the image information is preserved. Entropy is a statistical measure of randomness that can be used to characterize the texture of the input image [22]. In order to preserve information the difference in entropy between the original image and the enhanced image must be minimum. The mean square error in the pixel count for the calculation of ejection fraction and the difference in the entropy between the original image and enhanced image are less for the proposed method as compared to CLAHE method. Though the performance comparison in Table II indicates an improvement in contrast for the histogram equalization method, it fails to preserve the information content of the original image as evidenced by the values of MSE and entropy difference, which is very critical in the case of medical images. Using the proposed method, an improvement in contrast is obtained by preserving the information content of the original image.

TABLE II: PERFORMANCE COMPARISON

\begin{tabular}{cccc}
\hline \hline Measures & $\begin{array}{c}\text { Histogram } \\
\text { Equalization }\end{array}$ & CLAHE & $\begin{array}{c}\text { Proposed } \\
\text { Method }\end{array}$ \\
\hline MSE & 0.0069 & 0.000028 & 0.0000023 \\
Entropy difference & 1.2578 & 0.3356 & 0.0631 \\
$\begin{array}{c}\text { Contrast } \\
\text { improvement }\end{array}$ & 0.24 & 0.07 & 0.15 \\
\hline \hline
\end{tabular}

The proposed method, which uses an adaptive neighbourhood for the operation of structuring element, is also compared with the method which uses the same morphological operations and a fixed neighbourhood. For a fixed neighbourhood of smaller size the information content is preserved but the amount of contrast enhancement is very less. Fig. 12 shows the comparison between the proposed adaptive neighbourhood method and fixed neighbourhood method in enhancing contrast.

A good amount of contrast enhancement is achieved using a larger neighbourhood but with a variation in the information content. Fig. 13 shows a comparison between the proposed adaptive neighbourhood method and fixed neighbourhood method in preserving information. The entropy difference (information difference) is less for the proposed method as compared to the fixed neighbourhood method. Using adaptive selection of size of the structuring element a considerably good amount of contrast enhancement is achieved while preserving the information content as indicated by the MSE value and entropy difference value.

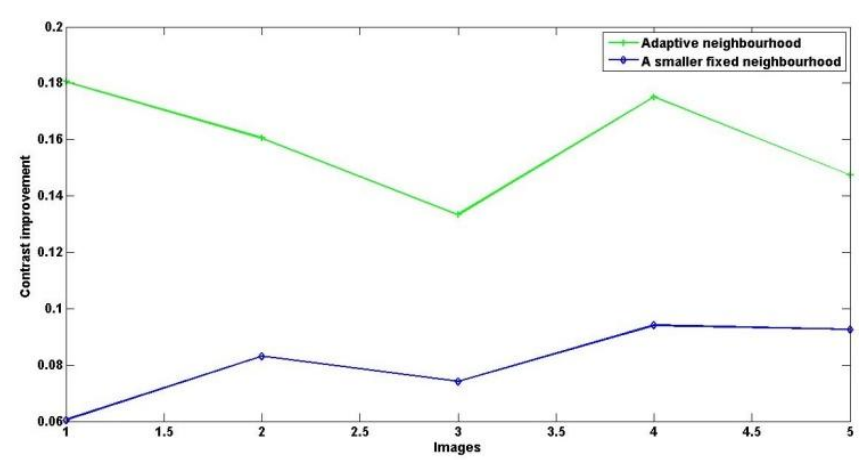

Fig. 12. Comparison of contrast improvement.

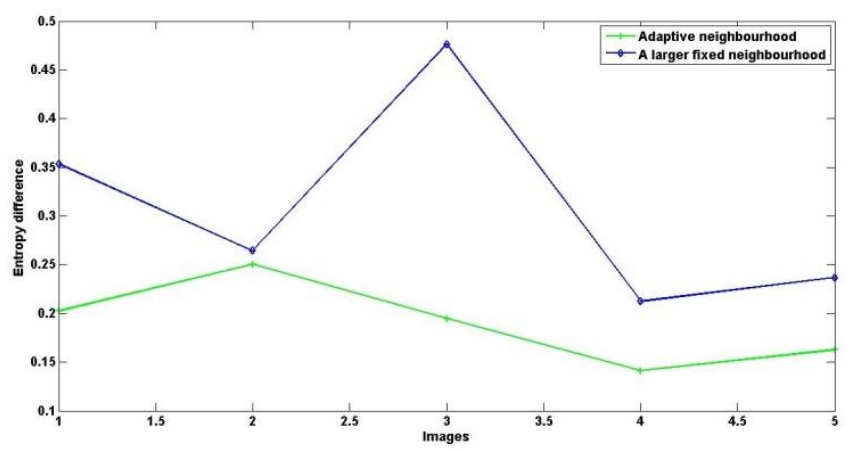

Fig. 13. Comparison of entropy difference.

\section{CONCLUSION}

In this paper we present a novel enhancement method for cardiac single photon emission computed tomography images. The proposed method operates in three major steps. First, the image is represented in a suitable color space and chrominance channels are separated. The type of the image region is subsequently analyzed and the size of the structuring element is selected. As a final step, the morphological operation is carried out in two stages. Since the size of the structuring element is dependent on the image region, this approach helps to meet the enhancement requirements of different image regions. Performance evaluations of the proposed method with respect to improvement in contrast and retention of original image information are executed on real cardiac images. The effectiveness of the method against the existing comparable techniques is studied. The experimental results prove that the method enhances nuclear images retaining image data integrity, recording an improvement over the existing techniques quantitatively.

\section{ACKNOWLEDGMENT}

The authors would like to thank Dr. Kuruvila Varkey, Nuclear Medicine Physician for his valuable comments on the results and for all his supports.

\section{REFERENCES}

[1] L. Koller, "The evolution of medical imaging technologies: Electric meat and the physicians shifting gaze," Ea Journal of Medical Humanities and Social Studies of Science and Technology, vol. 2, no. 3, pp. 1-22, April 2011. 
[2] V. Dilsizian and R. Taillefer, "Journey in evolution of nuclear cardiology: will there be another quantum leap with the f-18 labeled myocardial perfusion tracers?" Journal of American College of Cardiology, vol. 5, no. 12, pp. 1269-1284, December 2012.

[3] P. G. Camici and O. E. Rimoldi, "The clinical value of myocardial blood flow measurement," The Journal of Nuclear Medicine, vol. 50, no. 7, pp. 1076-1087, July 2009.

[4] A. Rahmim and H. Zaidi, "Pet versus spect: Strengths, limitations and challenges," Nuclear Medicine Communications, vol. 29, pp. 193-207, 2008.

[5] M. F. Di Carli and R. Hachamovitch, "Should pet replace spect for evaluating cad? The end of the beginning," Journal of Nuclear Cardiology, vol. 13, pp. 2-7, 2006.

[6] H. Wang and S. Ong, "Automated extraction of nuclear boundaries," in Proc. 2nd International Biomedical Engineering Days, 1998, pp. $85-87$.

[7] D. Falk, D. M. Rubin, and T. Marwala, "Enhancement of noisy planar nuclear medicine images using mean field annealing," International Federation for Medical and Biological Engineering Proceedings, vol. 4, no. 14, pp. 2415-2418, 2006.

[8] C. X. Wang, L. Small, W. E. Snyder, and R. Williams, "Edge detection in gated cardiac nuclear medicine images," in Proc. IEEE 7th Symposium on Computer Based Medical Systems, 1994, pp. 28-33.

[9] A. L. Pogam, H. Hanzouli, M. Hatt, C. C. L. Rest, and D. Visvikis, "Denoising of pet images by combining wavelets and curvelets for improved preservation of resolution and quantitation," Medical Image Analysis, Science Direct, vol. 17, p. 877891, 2013.

[10] G. Jammal and A. Bijaoui, "Multiscale image restoration for photon imaging systems," in Proc. IEEE International Conference on Acoustics and Speech Signal Processing, 1999, vol. 6, pp. 3401-3409.

[11] N. S. Arikidis, J. Kalatzis, S. Tsantis, V. Prassopoulos, and D. Cavouras, "Suppression of high frequency noise from scintigraphic images using the discrete wavelet transform," in Proc. Third European Symposium in Biomedical Engineering and Medical Physics, August 2002.

[12] K. Nawres, H. Kamel, and E. Noureddine, "Image denoising using wavelets: A powerful tool to overcome some limitations in nuclear imaging," in Proc. 2nd International Conference on Information and Communication Technologies: From Theory to Applications, 2006, pp. 1114-1118

[13] R. Mabrouk, W. Barhoumi, and R. B. Salah, "A novel approach for temporal covariance analysis of gated cardiac studies in scintigraphic images," in Proc. 2nd International Conference on Information and Communication Technologies: From Theory to Applications, April 2006, pp. 1812-1817.

[14] P. Brigger, S. L. Bacharach et al., "Segmentation of gated ti-spect images and computation of ejection fraction: A different approach," Journal of Nuclear Cardiology, vol. 6, no. 3, pp. 286-297, 1998.

[15] I. Mbazaa, N. Gribaa, N. Khlifa, and K. Hamroun, "A framework for nuclear image enhancement based on the anscomb transform and the bayesian thresholding," in Proc. 6th International Multi-conference on Systems, Signals and Devices, March 2009, pp. 1-6.

[16] J. Serra, Image Analysis and Mathematical Morphology Volume 1 and 2: Theoretical Advances, New York: Academic Press, 1988

[17] N. M. Sasi and V. K. Jayasree, "Edge detection in cardiac nuclear images," International Journal of Electronics and Communication Engineering, vol. 2, no. 2, pp. 53-56, May 2013.

[18] C. Poynton, A Technical Introduction to Digital Video, New York: Wiley, 1996

[19] A. Michelson, Studies in Optics, U. of Chicago Press, 1927.

[20] E. D. Pisano, S. Zong, B. M. Hemminger, M. De Luca, R. E. Johnstone, and S. M. Pizer, "Contrast limited adaptive histogram equalisation image processing to improve the detection of simulated spiculations in dense mammograms," Journal of Digital Imaging, vol. 11, no. 4, pp. 193-200, 1998.

[21] N. M. Sasi and V. K. Jayasree, "Contrast limited adaptive histogram equalization for the qualitative enhancement of myocardial perfusion images," Engineering Scientific Research Publishing, vol. 10, no. B, pp. 326-331, October 2013.

[22] Y. L. D.-Y. Tsai and E. Matsuyama, "Information entropy measure for evaluation of image quality," Journal of Digital Imaging, vol. 21, no. 3 , pp. 338-347, 2008.

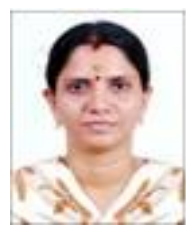

Neethu M. Sasi received the B.Tech degree in electronics and communication from Mahatma Gandhi University, India, with distinction and the M.Tech degree in signal processing from National Institute of Technology, Calicut, India, with gold medal in 2010. She is currently pursuing the $\mathrm{PhD}$ degree from the Department of Electronics, Govt. Model Engineering College, Thrikkakkara, Cochin University of Science and Technology, India. Since 2008 she has been dedicated to the design and development of signal and image processing systems. She developed a low complexity space time adaptive system algorithm for sonar detection and presented it in 2010 IEEE Sensor Array and Multichannel Signal Processing Workshop conducted in Jerusalem. As an IEEE member for the past 6 years, her research interests include signal processing, image processing and use of adaptive techniques in image processing.

Jayasree V. K. received the M.Tech degree in biomedical engineering from Indian Institute Technology, Chennai, India in 1998 and the $\mathrm{PhD}$ degree from Cochin University of Science and Technology, India in 2009.

Currently she is an associate professor and the head of the Department of Electronics, Govt. Model Engineering College, Cochin University of Science and Technology, India. Her research interests include biomedical image processing and optical communication techniques. 